\title{
Association between initial vascular access and survival in hemodialysis according to age
}

\author{
Ha Yeon Kim, Eun Hui Bae, Seong Kwon Ma, and Soo Wan Kim
}

Department of Internal Medicine, Chonnam National University Medical School, Gwangju, Korea

Received: January 18, 2017

Revised : February 28, 2017

Accepted: June 19, 2017

\section{Correspondence to}

Soo Wan Kim, M.D.

Department of Internal Medicine, Chonnam National University

Medical School, 42 Jebongro,

Gwangju 61469, Korea

Tel: $+82-62-220-6271$

Fax: +82-62-225-8578

E-mail: skimw@chonnam.ac.kr
Background/Aims: This study aims to demonstrate whether the association between initial vascular access and mortality among hemodialysis patients varies by age.

Methods: We conducted a retrospective study that included 2,552 patients who started hemodialysis. Vascular access was divided into three categories: percutaneous catheter, tunneled cuffed catheter, and arteriovenous (AV) access.

Results: Survival rates for patients who received a central venous catheter, such as percutaneous or tunneled cuffed catheter, aged 65 to 74 years and those $\geq 75$ years were reduced, but not for those aged $<65$ years (log-rank test; $p<0.001, p=$ 0.007 , and $p=0.278)$. After fully adjusting for potential confounding factors in the patients aged $<65$ years, percutaneous and tunneled cuffed catheter were not associated with 5-year mortality. On the other hand, for patients aged 65 to 74 or $\geq$ 75 years, percutaneous catheter and tunneled cuffed catheter were associated with higher 5-year mortality rates. As age increased, the conversion rate from central venous catheter, including percutaneous catheter and tunneled cuffed catheter, to $\mathrm{AV}$ access decreased (94.1\%, 90.5\%, and $80.3 \%$ for patients aged $<65,65$ to 74 , and $\geq$ 75 years, respectively; $p<0.001)$.

Conclusions: In patients aged $\geq 65$ years, initial vascular access was associated with long-term mortality. We suggest that a "fistula first" strategy is superior for elderly patients and demonstrates that it is desirable to change to AV access, and not maintain an initial central vascular catheter.

Keywords: Vascular access; Age; Hemodialysis

\section{INTRODUCTION}

Proper vascular access is essential to provide efficient dialysis therapy. Vascular access complications remain the leading cause of morbidity in hemodialysis patients and are a major contributor to high healthcare costs. The central venous catheter has been linked to greater risk of infection, hospitalization, and mortality, and has been known to compromise dialysis adequacy [1-7]. As a result, arteriovenous fistula (AVF) use is strongly recommended by many guidelines [8-12].
Over the years, there has been marked growth in the use of dialysis to treat elderly patients. In Korea, patients aged $\geq 65$ years increased to $37.5 \%$ of the total number of dialysis patients [13]. There is, however, controversy about the benefits of AVF compared with the use of central vascular catheter, especially for elderly or very old-aged patients $[14,15]$. Treatment challenges among elderly patients include comorbid conditions and non-maturing fistula [16]. In addition, elderly patients have notable differences in kidney function, timing and initiation of dialysis, quality-of-life issues, and life ex- 
pectancy [17]. However, clinical practice guidelines are rarely age-specific and do not address issues specific to the well-being of elderly patients. Despite established guidelines, a very high proportion of patients start dialysis with a central venous catheter because of late referral to a nephrology team $[18,19]$ and the increased use of hemodialysis for acute kidney injury (AKI).

The association between initial vascular access for hemodialysis and mortality is strongly linked, and AVF has been shown to be of benefit in a survival analysis of hemodialysis patients [20]. However, a previous study suggests that the association between the use of a central venous catheter and mortality might be the result of AKI itself, and that mortality for patients implanted with a central venous catheter may be confounded without considering the influence of an acute dialysis start $[21,22]$. There was also a selection bias because patients receiving the central venous catheter were in worse vascular condition or had more comorbidities. Despite careful adjustment for various factors, it remains dif- ficult to account for bias. Most patients who begin hemodialysis via central venous catheter change to arteriovenous (AV) access, including AVF and AV graft, in the long run. However, physicians also considered conversions in light of the patient's age, remaining life expectancy, and comorbid condition. The aim of the present study was to demonstrate whether the association between initial vascular access and all-cause mortality among hemodialysis patient might vary at different ages and conversion rate might differ according to age.

\section{METHODS}

\section{Ethics statement}

This study was approved by the Institutional Review Board of Chonnam National University Hospital, Gwangju, Korea (CNUH-2012-108). The study was performed in accordance with the Helsinki Declaration of 1975, as revised in 2000. The Institutional Review Board
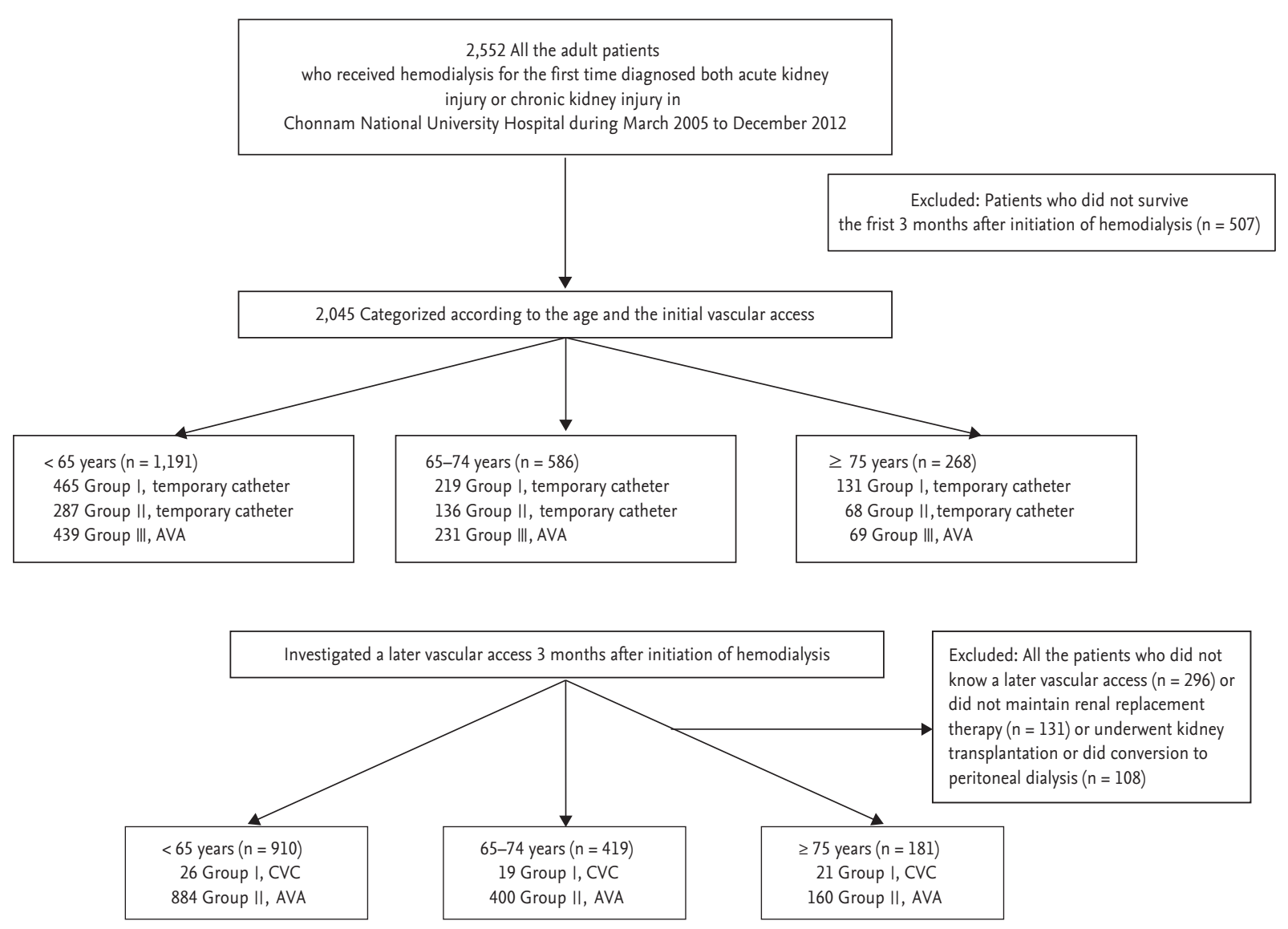

Figure 1. Outline of the study design and exclusion criteria. AVA, arteriovenous access; CVC, central venous catheter. 
waived the need for consent given the retrospective design of the project. The mortality data were determined from government death records from Statistics Korea.

\section{Subjects}

We conducted a retrospective study of 2,552 patients who started hemodialysis at Chonnam National University Hospital between March 2005 and December 2012. Subsequently, 507 patients who did not survive the first 3 months after initiation of hemodialysis were excluded because outcomes may be influenced by a patient's health status before hemodialysis commencement rather than type of vascular access. For the final study, 2,045 patients were enrolled (Fig. 1). Patients in the survey were diagnosed with AKI or chronic kidney disease and underwent hemodialysis treatment for more than three consecutive months. We presupposed that the bias of an acute dialysis start or risk of AKI itself could be reduced by this exclusion. Hemodialysis was conducted via central venous catheter or AV access. The central venous catheter is associated with percutaneous catheter and tunneled cuffed catheter; and AV access is associated with AVF and AV graft. Three months after hemodialysis initiation, vascular access was reinvestigated because many patients underwent an AV access operation for long-term hemodialysis. The following patients were excluded: those who did not receive later vascular access $(n=296)$, those who did not maintain renal replacement therapy $(n=131)$, and those who underwent kidney transplantation or conversion to peritoneal dialysis $(n=108)$. Patients were subdivided into three age groups $(<65,65$ to 74 , and $\geq 75$ years). Demographic, clinical, and laboratory parameters, including creatinine, hemoglobin, and albumin levels, and treatment data were obtained from electronic patient records. The estimated glomerular filtration rate (eGFR) was calculated using the Chronic Kidney Disease Epidemiology Collaboration (CKD-EPI) formula: $\mathrm{mL} / \mathrm{min} / 1.73 \mathrm{~m}^{2}=141 \times$ minimum (creatinine $/ \mathrm{k}$, $1)^{\alpha} \times$ maximum (creatinine $\left./ \mathrm{k}, 1\right)^{-1.209} \times 0.993^{\text {age }} \times 1.018$ (if, female) $\times 1.159$ (if, black), where $\kappa$ is 0.7 for women and 0.9 for men, and $\alpha$ is -0.329 for women and -0.411 for men [23]. The patient's vascular access was divided into three categories (percutaneous catheter, tunneled cuffed catheter, and AV access). Follow-up for the whole group of patients was $35.2 \pm 21.72$ months.

\section{Statistical analysis}

Continuous variables with normal distributions are presented as means \pm standard deviation and were compared using one-way ANOVA. Pearson chi-squared test was used to evaluate differences between categorical variables. Survival curves were compared by log-rank tests. Multivariable Cox regression analysis was applied to adjust for the baseline factors: e.g., age, gender, diabetes, heart failure, cardioprotective medication (e.g., $\beta$-blocker, calcium channel blocker, angiotensin converting enzyme inhibitor, angiotensin receptor antagonist medication) and hemodialysis vascular access. Cox proportional hazards regression analysis was performed to evaluate the prognostic significance of initial vascular access for mortality in hemodialysis patients. All statistical tests were performed using the SPSS version 18.0 (SPSS Inc., Chicago, IL, USA). $p$ values $<0.05$ were considered significant.

\section{RESULTS}

\section{Baseline characteristics}

A total of 2,045 patients (59\% male) were included in the study. The mean age was $58.5 \pm 14.5$ years. Three age categories were evaluated for patients who initiated hemodialysis. At baseline, $58.2 \%(\mathrm{n}=1,191)$ of the patients were $<65$ years old, $28.7 \%(n=586)$ were 65 to 74 years old, and $13.1 \%(n=268)$ were $\geq 75$ years old. The percentage of hemodialysis patient $\geq 65$ years was $41.8 \%$. Percutaneous catheter use accounted for $39.8 \%(n=815)$ of all incident hemodialysis patients, whereas tunneled cuffed catheter accounted for $24.0 \%(n=491)$ and AV access accounted for $36.1 \%(n=739)$. Table 1 shows the patients' baseline characteristics by vascular access type. In general, across all age categories, patients who were female and who had lower serum hemoglobin levels, lower serum albumin levels, increased white blood cell levels, increased C-reactive protein levels, and who used inotropic or vasopressor agents were more likely to receive a percutaneous catheter or tunneled cuffed catheter than AV access. Table 2 lists the baseline characteristics of the study population by age category. There was a trend toward increased percutaneous catheter or tunneled cuffed catheter use for older patients ( $\geq 75$ years) than those aged $<$ 65 years or those 65 to 74 years old $(p=0.001)$. There were 


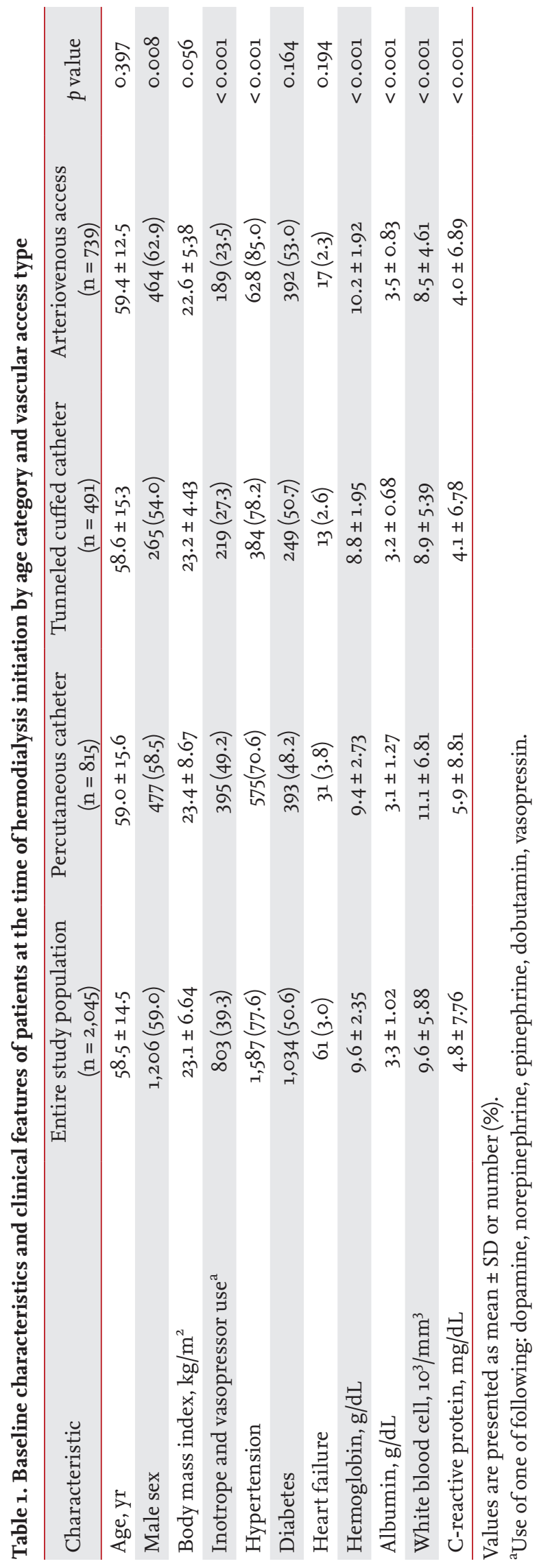

no differences in hemoglobin, sodium, or albumin levels among the groups. Although serum creatinine level decreased with increasing age, there was no difference in eGFR among the three groups.

\section{Initial vascular access-associated mortality by age group}

In all, 716 patients (35.0\%) died and overall mortality increased with age $(23.6 \%, 46.6 \%$, and $48.0 \%$ for patients aged $<65,65$ to 74 , and $\geq 75$ years, respectively; $p=0.001$ ). We investigated the impact of initial vascular access on 5-year mortality. Fig. 2 showed 5-year survival in patients starting hemodialysis according to age and vascular access, patients aged $\geq 65$ years with central venous catheter showed the lowest survival curve, whereas patients aged $<65$ years with AV access showed the highest survival curve (log-rank test; $p<0.001)$. Kaplan-Meier survival analysis showed that the survival rate for patients with central venous catheter aged 65 to 74 and $\geq 75$ years was reduced, but not for those aged $<65$ years (log-rank test; $p<0.001, p=0.007$, and $p=0.278$, respectively) (Fig. 3).

To determine risk factors for 5-year mortality in patients starting hemodialysis, Cox proportional analysis was performed (Table 3). In adjusted Cox proportional analysis; age per years (hazard ratio [HR], 1.04; 95\% confidence interval [CI], 1.03 to 1.05); diabetes (HR, 1.88; 95\% CI, 1.59 to 2.21); albumin < $3.5 \mathrm{~g} / \mathrm{dL}$ (HR, 1.43; 95\% CI, 1.22 to 1.68); and hemoglobin < 10.0 g/dL (HR, 1.17; 95\% CI, 1.00 to 1.37). Compared with AV access, percutaneous catheter (HR, 1.78; 95\% CI, 1.44 to 2.21 ) and tunneled cuffed catheter (HR, 1.64; 95\% CI, 1.36 to 1.98) were significant risk factors for 5 -year mortality in patients

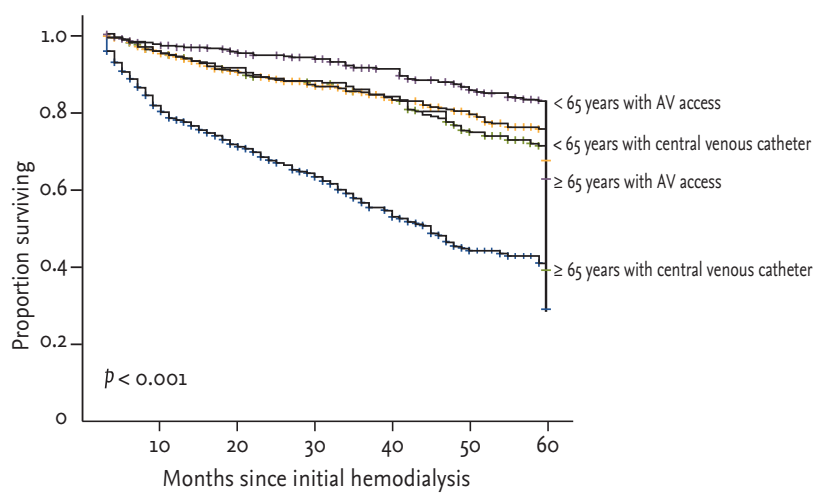

Figure 2. Kaplan-Meier survival analysis according to age group and vascular access type. AV, arteriovenous. 
starting hemodialysis.

Table 4 showed the differences in 5-year mortality in patients starting hemodialysis according to vascular access types and age group. The overall 5-year mortality in all subjects was $31.0 \%(634 / 2,045)$. The 5 -year mortality was increased in the order of AV access, tunneled cuffed catheter and percutaneous catheter, and also increased with age. An increased risk for death in the hemodialysis patients with percutaneous catheter was more pro- nounced in older subjects: in patients aged $\geq 75$ years (HR, 2.78; 95\% CI, 1.92 to 4.03); in patients aged 65 to 74 years (HR, 2.43; 95\% CI, 1.82 to 3.23) as compared with patients aged $<65$ years (HR, 1.18; 95\% CI, 0.89 to 1.56), the value of $p$ for interaction between vascular access and age group is $<0.001$. Table 5 lists associations between vascular access and 5-year mortality in increasingly adjusted models. Compare to AV access, percutaneous catheter and tunneled cuffed catheter were risk factors
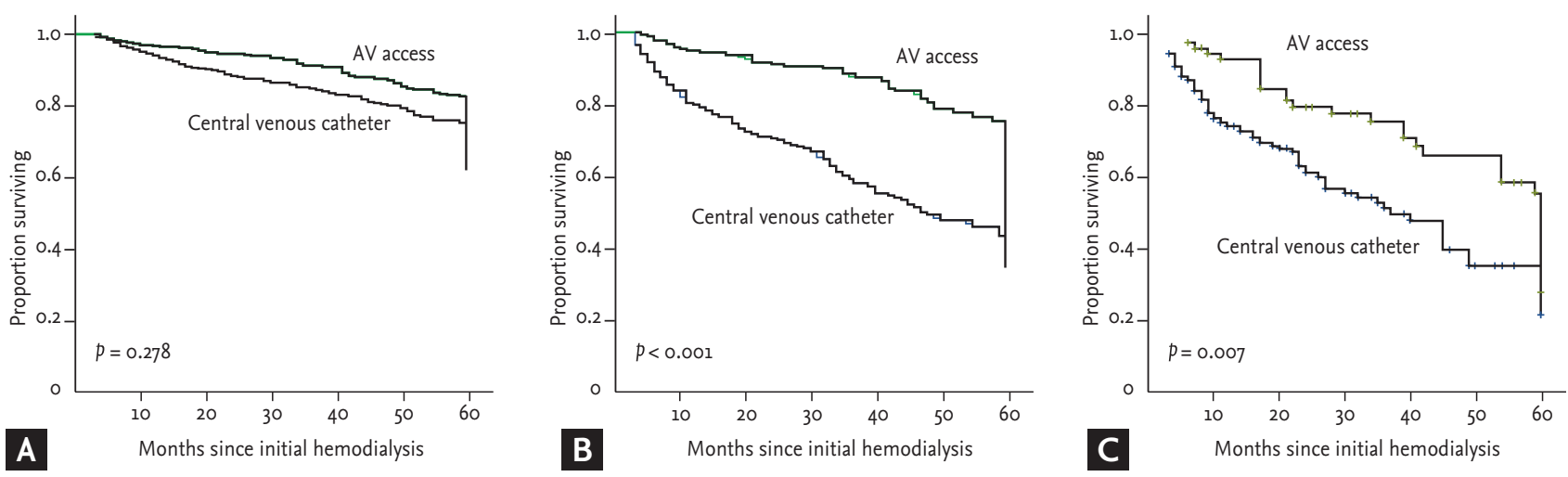

Figure 3. Kaplan-Meier survival analysis according to initial vascular access for the three age categories: (A) $<65$ years, (B) 65 to 74 years, and $(\mathrm{C}) \geq 75$ years. AV, arteriovenous.

Table 2. Baseline characteristics and clinical features of patients according to age category

\begin{tabular}{|c|c|c|c|c|}
\hline Characteristic & $<65$ Years $(n=1,191)$ & $65-74$ Years $(n=586)$ & $\geq 75$ Years $(n=268)$ & $p$ value \\
\hline Age, yr & $49.6 \pm 11.05$ & $69.3 \pm 2.80$ & $79.0 \pm 3.53$ & $<0.001$ \\
\hline Male sex & $783(61.3)$ & $336(56.0)$ & $153(54.8)$ & 0.031 \\
\hline Body mass index, $\mathrm{kg} / \mathrm{m}^{2}$ & $23.2 \pm 7.83$ & $23.0 \pm 3.74$ & $22.3 \pm 3.79$ & 0.205 \\
\hline Late referral & $603(47 \cdot 3)$ & $277(46.2)$ & $153(54.8)$ & 0.070 \\
\hline \multicolumn{5}{|l|}{ Past history } \\
\hline Hypertension & $944(74.0)$ & $503(83.7)$ & $231(82.8)$ & $<0.001$ \\
\hline Diabetes & $599(47 \cdot 0)$ & $375(62.4)$ & $117(41.9)$ & $<0.001$ \\
\hline Heart failure & $31(2.4)$ & $18(3.0)$ & $14(5.0)$ & 0.067 \\
\hline \multicolumn{5}{|l|}{ Arteriovenous access type $\mathrm{a}^{\mathrm{a}}$} \\
\hline Percutaneous catheter & $465(39.0)$ & $219(37.4)$ & $131(48.9)$ & 0.001 \\
\hline Tunneled cuffed catheter & $287(24.1)$ & $136(23.2)$ & $68(25.4)$ & 0.001 \\
\hline Arteriovenous access & $439(36.9)$ & $231(39.4)$ & $69(25 \cdot 7)$ & 0.001 \\
\hline Conversion rate & 94.1 & 90.5 & 80.3 & $<0.001$ \\
\hline \multicolumn{5}{|l|}{ Chemistry profile } \\
\hline Hemoglobin, g/dL & $9.5 \pm 2.51$ & $9.6 \pm 2.04$ & $9.4 \pm 2.14$ & 0.710 \\
\hline Albumin, g/dL & $3 \cdot 3 \pm 1.19$ & $3.3 \pm 0.64$ & $3.2 \pm 0.60$ & 0.091 \\
\hline Serum creatinine, $\mathrm{mg} / \mathrm{dL}$ & $9.0 \pm 6.49$ & $7.0 \pm 3.49$ & $6.2 \pm 3.43$ & $<0.001$ \\
\hline eGFR, $\mathrm{mL} / \mathrm{min} / 1.73 \mathrm{~m}^{2}$ & $10.7 \pm 18.67$ & $11.3 \pm 21.36$ & $12.0 \pm 14.14$ & 0.519 \\
\hline
\end{tabular}

Values are presented as mean $\pm \mathrm{SD}$ or number (\%).

${ }^{\mathrm{a}}$ Arteriovenous fistula or arteriovenous graft. 
Table 3. Cox proportional analysis for 5-year mortality in all cohort population

\begin{tabular}{lrr}
\hline Variable & HR $(95 \%$ CI $)$ & $p$ value \\
\hline Age, yr & $1.04(1.03-1.05)$ & $<0.001$ \\
Male sex & $1.12(0.96-1.31)$ & 0.144 \\
Diabetes & $1.88(1.59-2.21)$ & $<0.001$ \\
Congestive heart failure & $1.53(0.98-2.39)$ & 0.060 \\
Cardioprotective medication ${ }^{\mathrm{a}}$ & $1.16(1.00-1.36)$ & 0.051 \\
Albumin <3.5 g/dL & $1.43(1.22-1.68)$ & $<0.001$ \\
Hemoglobin $<10.0$ g/dL & $1.17(1.00-1.37)$ & 0.045 \\
Percutaneous catheter (ref. AV access) & $1.78(1.44-2.21)$ & $<0.001$ \\
Tunneled cuffed catheter (ref. AV access) & $1.64(1.36-1.98)$ & $<0.001$ \\
\hline
\end{tabular}

HR, hazard ratio; CI, confidence interval; AV, arteriovenous.

${ }^{\mathrm{a}}$ One of following: $\beta$-blocker, calcium channel blocker, angiotensin converting enzyme inhibitor, angiotensin receptor antagonist medication.

Table 4. Hazard ratios for the mortality in subgroups

\begin{tabular}{|c|c|c|c|c|c|c|c|}
\hline \multirow[b]{2}{*}{ Variable } & \multicolumn{3}{|c|}{ No. of the deaths/patients (\%) } & \multicolumn{3}{|c|}{$\operatorname{HR}(95 \% \mathrm{CI})$} & \multirow{2}{*}{$\begin{array}{l}p \text { value for } \\
\text { interaction }\end{array}$} \\
\hline & $\begin{array}{c}\text { Percutaneous } \\
\text { catheter }\end{array}$ & $\begin{array}{c}\text { Tunneled } \\
\text { cuffed catheter }\end{array}$ & AV access & $\begin{array}{c}\text { Percutaneous } \\
\text { catheter }\end{array}$ & $\begin{array}{c}\text { Tunneled } \\
\text { cuffed catheter }\end{array}$ & AV access & \\
\hline Age group, yr & $319 / 815(39.1)$ & $175 / 491(35.6)$ & $140 / 739(18.9)$ & - & - & - & \\
\hline$<6_{5}$ & $95 / 465(20.4)$ & $60 / 287(21.0)$ & $78 / 439(17.8)$ & $1.18(0.89-1.56)$ & $1.28(0.92-1.78)$ & Reference & \\
\hline $65-74$ & $127 / 219(58.0)$ & $69 / 136(50.7)$ & $45 / 231(19.5)$ & $2.43(1.82-3.23)$ & $2.27(1.64-3.13)$ & Reference & \\
\hline$\geq 75$ & $97 / 131(74.0)$ & $46 / 68(67.6)$ & $17 / 69(24.6)$ & $2.78(1.92-4.03)$ & $1.92(1.23-2.98)$ & Reference & \\
\hline
\end{tabular}

HR, hazard ratio; CI, confidence interval; AV, arteriovenous.

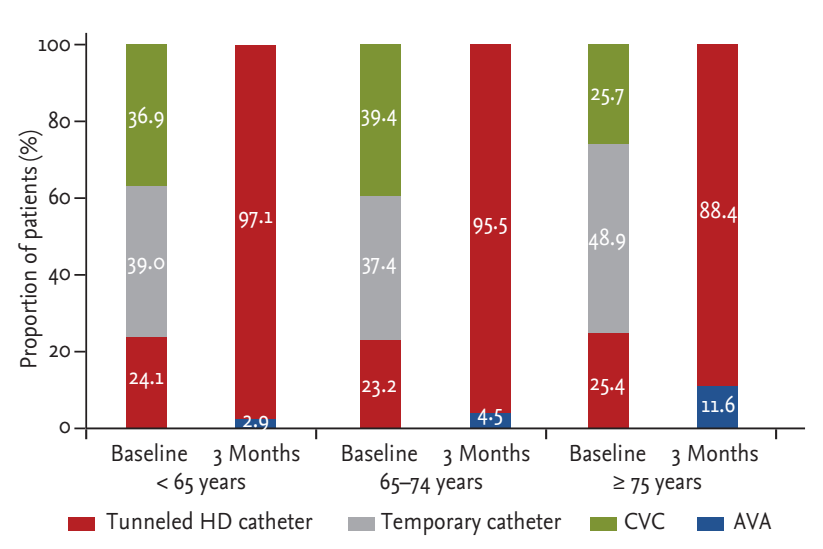

Figure 4. The proportion of vascular access type at baseline (i.e., start of hemodialysis) and 3 months after hemodialysis initiation for the three age categories. HD, hemodialysis; CVC, central venous catheter; AVA, arteriovenous access.

for patients aged 65 to 74 years and those $\geq 75$ years. After fully adjusting for potential confounding factors including vascular conversion, patients aged $<65$ years, percutaneous and tunneled cuffed catheter, were not as- sociated with 5-year mortality ([HR, 1.28; 95\% CI, o.89 to 1.82] and [HR, 1.04; 95\% CI, 0.74 to 1.45], respectively). On the other hand, for patients aged 65 to 74 and $\geq 75$ years, percutaneous catheter ([HR, 1.97; 95\% CI, 1.35 to 2.74] and [HR, 1.93; 95\% CI, 1.35 to 2.74], respectively) and tunneled cuffed catheter ([HR, 1.89; 95\% CI, 1.10 to 3.26] and [HR, 1.34 ; $95 \%$ CI, 1.05 to 2.26 ], respectively) were significantly associated with 5 -year mortality.

\section{Vascular access conversion after 3 months by age group}

Conversion rate means the proportion of patients who changed their vascular access type from central venous catheter to AV access or who underwent AV access creation. As age increased, the conversion rate decreased (94.14\%, 90.54\%, and $80.37 \%$ for each age group; $p=$ 0.001) (Table 2).

Fig. 4 shows the proportion of vascular access both at the time of hemodialysis initiation and 3 months after hemodialysis initiation among the three age categories. 


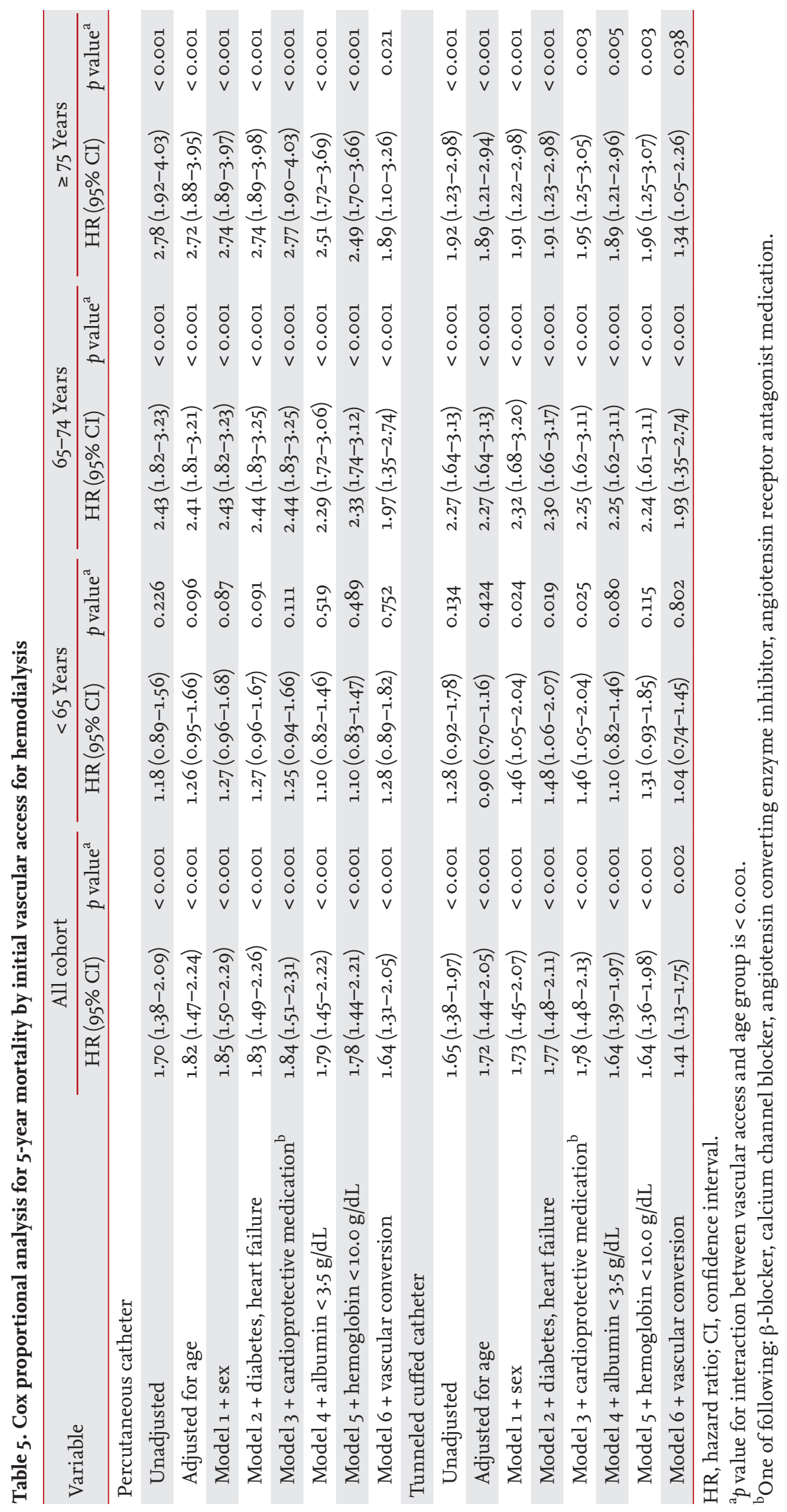


Most patients who initiated hemodialysis therapy using a central venous catheter exchanged vascular access to $\mathrm{AV}$ access (94.1\%) (Table 2), with $2.9 \%$ remaining on a central venous catheter 3 months later in patients aged $<65$ years (Fig. 4). Use of the central venous catheter reduced from a proportional value of $63.1 \%$ (percutaneous catheter, $39.0 \%$ + tunneled cuffed catheter, $24.1 \%)$ to $2.9 \%$ in those $<65$ years old, from $60.6 \%$ (percutaneous catheter, $37.4 \%$ + tunneled cuffed catheter, $23.2 \%$ ) to $4.5 \%$ in those 64 to 74 years old, and from $74.3 \%$ (percutaneous catheter, $48.9 \%$ + tunneled cuffed catheter, $25.4 \%$ ) to $11.6 \%$ in those $\geq 75$ years old. On the other hand, AV access increased among all three groups from a proportional value of $36.9 \%$ to $97.1 \%$ ( $<65$ years), $39.4 \%$ to $95.5 \%$ (64 to 74 years), and $25.7 \%$ to $88.4 \%$ ( $\geq 75$ years). It is suggested that much older patients better maintained central venous catheter 3 months after hemodialysis initiation and had a lower conversion rate of AV access.

\section{DISCUSSION}

Our results confirm that elderly patients $\geq 75$ years are less likely to have AV access, and more likely to receive a central venous catheter. The initial vascular access in elderly patients was mainly via a percutaneous or tunneled cuffed catheter; $48.9 \%$ of patients $\geq 75$ years initiated dialysis with a percutaneous catheter, $25.4 \%$ with a tunneled cuffed catheter, and $25.7 \%$ with AV access. Unplanned dialysis initiation was defined as starting dialysis without functional vascular access. More prevalent dialysis initiation with percutaneous catheters suggests that elderly patients are more likely to start dialysis in an unplanned manner, which may be associated with greater mortality rates in incident dialysis patients $[24,25]$. Results of the present study correspond with the features of unplanned hemodialysis in that patients with percutaneous catheters were more likely to use inotrope and vasopressor agents and have increased inflammatory or infectious markers such as leukocytosis or an elevated level of C-reactive protein.

The present study shows an age-related increase in mortality among all patients who start hemodialysis. We postulate that patients aged $\geq 65$ years had a lesser proportion of AV access and more central venous catheter, which linked to unplanned hemodialysis at baseline and the 3-month follow-up assessment. The elderly often have vascular pathologies such as calcification, atherosclerosis, or endothelial dysfunction $[26,27]$. These vascular changes may have adverse effects on the creation of a fistula and fistula maturation [28], and may be an additional factor in reduced first AV access for elderly patients who start hemodialysis. The present study also showed reduced AV access in patients $\geq 65$ years at both baseline and 3 months after hemodialysis initiation.

In this observational study of consecutive incident dialysis patients, we attempted to determine whether initial vascular access type would have an effect on survival comparisons among different age groups. In contrast to previous studies, patients younger than 65 years showed no more risk with a central venous catheter than those receiving AV access. This might be caused by timely conversion to AV access and the exclusion of death, which occurred within 3 months after starting hemodialysis.

We assumed that physicians tend to maintain initial vascular access via percutaneous catheter in patients $\geq$ 65 years because elderly patients have more comorbidity, which may be caused by an increased risk of complications with procedures or lowered life expectancy. Indeed, the conversion rate showed a difference among groups according to age categories. The rate of conversion decreased with advancing age, and was lowest in patients aged $\geq 75$ years. These findings are in agreement with previous studies in which conversion from a catheter to permanent vascular access was associated with an adjusted mortality HR of 0.69 (95\% CI, 0.55 to $0.85)$ [29]. This implies that vascular access for hemodialysis should first consider AV access or conversion to AV access in patients of all ages.

This study has several limitations. First, vascular access associated with mortality in patients with hemodialysis has a higher clinical impact on short-term mortality. However, we excluded patients who died within 3 months of hemodialysis initiation; therefore, the shortterm morality could not be further analyzed. Second, there is a possibility of being misunderstood that is no association between vascular access type and long-term mortality in the patients $<65$ years with hemodialysis. Because most of them had switched AV access, its significance might not be apparent. Last, because of the limitation of retrospective study, AV access is not distinguished by fistula and graft. Since grafts are known to 
have more vascular complications such as an increases of thromboembolic events and infection risk due to endothelial dysfunction, further analysis is needed in the future.

In conclusion, in patients $<65$ years, initial vascular access is not associated with long-term mortality, because most of patients $<65$ years had conversion from a central venous catheter to AV access within 3 months. Thus it is more reasonable to start hemodialysis immediately via an available central venous catheter for an emergent medical condition, rather than delay dialysis owing to vascular access. In patients aged $\geq 65$ years, initial vascular access was associated with long-term mortality. "Fistula first" remains a superior strategy for elderly patients and demonstrates it is desirable to change AV access, not maintain the initial central vascular catheter.

\section{KEY MESSAGE}

1. Survival rates for patients who received a central venous catheter aged 65 to 74 years and those $\geq$ 75 years were reduced, but not for those aged < 65 years.

2. As age increased, the conversion rate from central venous catheter to arteriovenous (AV) access decreased.

3. We suggest that a "fistula first" strategy is superior for elderly patients and demonstrates that it is desirable to change to AV access, and not maintain an initial central vascular catheter.

\section{Conflict of interest}

No potential conflict of interest relevant to this article was reported.

\section{Acknowledgments}

This research was supported by the Basic Science Research Program through the National Research Foundation of Korea (NRF) funded by the Ministry of Science, ICT and Future Planning(MSIP)(2015R1D1A1A01061037), by Basic Science Research Program through the NRF funded by the MSIP (2016R1A2B4007870), by the Pioneer Research Center Program through the NRF funded by the MSIP (2014M3C1A3053036), and by a grant of the Korea Health Technology R\&D Project through the
Korea Health Industry Development Institute (KHIDI), funded by the Ministry of Health and Welfare, Korea (HI14C2084), by the Bio \& Medical Technology Development Program of the NRF funded by the MSIP (2017M3A9E8023001), and by a grant of the Chonnam National University Hospital Biomedical Research Institute (CRI16013-1).

\section{REFERENCES}

1. Hoen B, Kessler M, Hestin D, Mayeux D. Risk factors for bacterial infections in chronic haemodialysis adult patients: a multicentre prospective survey. Nephrol Dial Transplant 1995;10:377-381.

2. Combe C, Pisoni RL, Port FK, et al. Dialysis outcomes and practice patterns study: data on the use of central venous catheters in chronic hemodialysis. Nephrologie 2001;22:379-384.

3. Polkinghorne KR, McDonald SP, Atkins RC, Kerr PG. Vascular access and all-cause mortality: a propensity score analysis. J Am Soc Nephrol 2004;15:477-486.

4. Pastan S, Soucie JM, McClellan WM. Vascular access and increased risk of death among hemodialysis patients. Kidney Int 2002;62:620-626.

5. Oliver MJ, Rothwell DM, Fung K, Hux JE, Lok CE. Late creation of vascular access for hemodialysis and increased risk of sepsis. J Am Soc Nephrol 2004;15:19361942.

6. Moist LM, Trpeski L, Na Y, Lok CE. Increased hemodialysis catheter use in Canada and associated mortality risk: data from the Canadian Organ Replacement Registry 2001-2004. Clin J Am Soc Nephrol 2008;3:1726-1732.

7. Polkinghorne KR. Vascular access practice in hemodialysis: instrumental in determining patient mortality. Am J Kidney Dis 2009;53:359-362.

8. NKF-DOQI clinical practice guidelines for vascular access: National Kidney Foundation-Dialysis Outcomes Quality Initiative. Am J Kidney Dis 1997;30(4 Suppl 3):S150-S191.

9. Ethier JH, Lindsay RM, Barre PE, Kappel JE, Carlisle EJ, Common A. Clinical practice guidelines for vascular access: Canadian Society of Nephrology. J Am Soc Nephrol 1999;10 Suppl 13:S297-S305.

10. III. NKF-K/DOQI Clinical practice guidelines for vascular access: update 2000. Am J Kidney Dis 2001;37(1 Suppl 1):S137-S181. 
11. Allon M. Current management of vascular access. Clin J Am Soc Nephrol 2007;2:786-800.

12. Lok CE. Fistula first initiative: advantages and pitfalls. Clin J Am Soc Nephrol 2007;2:1043-1053.

13. Jin DC, Han JS. Renal replacement therapy in Korea, 2012. Kidney Res Clin Pract 2014;33:9-18.

14. Di Iorio BR, Bellizzi V, Cillo N, et al. Vascular access for hemodialysis: the impact on morbidity and mortality. J Nephrol 2004;17:19-25.

15. Duncan ND, Singh S, Cairns TD, et al. Tesio-Caths provide effective and safe long-term vascular access. Nephrol Dial Transplant 2004;19:2816-2822.

16. Lok CE, Allon M, Moist L, Oliver MJ, Shah H, Zimmerman D. Risk equation determining unsuccessful cannulation events and failure to maturation in arteriovenous fistulas (REDUCE FTM I). J Am Soc Nephrol 2006;17:32043212.

17. Moist LM, Lok CE, Vachharajani TJ, et al. Optimal hemodialysis vascular access in the elderly patient. Semin Dial 2012;25:640-648.

18. Pisoni RL. Vascular access use and outcomes: results from the DOPPS. Contrib Nephrol 2002;(137):13-19.

19. Mendelssohn DC, Kua BT, Singer PA. Referral for dialysis in Ontario. Arch Intern Med 1995;155:2473-2478.

20. Ravani P, Palmer SC, Oliver MJ, et al. Associations between hemodialysis access type and clinical outcomes: a systematic review. J Am Soc Nephrol 2013;24:465-473.

21. Tennankore KK, Soroka SD, Kiberd BA. The impact of an "acute dialysis start" on the mortality attributed to the use of central venous catheters: a retrospective cohort study. BMC Nephrol 2012;13:72.
22. Descamps C, Labeeuw M, Trolliet $\mathrm{P}$, et al. Confounding factors for early death in incident end-stage renal disease patients: role of emergency dialysis start. Hemodial Int 2011;15:23-29.

23. Levey AS, Stevens LA, Schmid CH, et al. A new equation to estimate glomerular filtration rate. Ann Intern Med 2009;150:604-612.

24. Lorenzo V, Martn M, Rufino M, Hernandez D, Torres A, Ayus JC. Predialysis nephrologic care and a functioning arteriovenous fistula at entry are associated with better survival in incident hemodialysis patients: an observational cohort study. Am J Kidney Dis 2004;43:999-1007.

25. Couchoud C, Moranne O, Frimat L, Labeeuw M, Allot V, Stengel B. Associations between comorbidities, treatment choice and outcome in the elderly with end-stage renal disease. Nephrol Dial Transplant 2007;22:3246-3254.

26. Garland JS, Holden RM, Groome PA, et al. Prevalence and associations of coronary artery calcification in patients with stages 3 to $5 \mathrm{CKD}$ without cardiovascular disease. Am J Kidney Dis 2008;52:849-858.

27. Hassan NA, D'Orsi ET, D'Orsi CJ, O'Neill WC. The risk for medial arterial calcification in CKD. Clin J Am Soc Nephrol 2012;7:275-279.

28. Khan FA, Vesely TM. Arterial problems associated with dysfunctional hemodialysis grafts: evaluation of patients at high risk for arterial disease. J Vasc Interv Radiol 2002; 13:1109-1114.

29. Bradbury BD, Chen F, Furniss A, et al. Conversion of vascular access type among incident hemodialysis patients: description and association with mortality. Am J Kidney Dis 2009;53:804-814. 